\title{
THE NECESSITY OF HARRIS' CONDITION FOR THE EXISTENCE OF A STATIONARY MEASURE
}

\author{
WILLIAM VEECH ${ }^{1}$
}

1. Introduction. Let $P$ be the matrix of transition probabilities for an irreducible transient Markov chain with state space the nonnegative integers. Harris obtains in [1] a sufficient condition for the existence of a positive vector solution to the equation

$$
a P=a .
$$

He feels that his condition is also close to being necessary, and the main purpose of this note is to prove this fact. ${ }^{2}$ In $\$ 4$ we present an unrelated result of some interest which we had mistakenly thought to be essential for Theorem 1.

2. Facts about transient chains. Recall that transience implies the sum

$$
Q=\sum_{n=0}^{\infty} P^{n}
$$

exists, while irreducibility guarantees to any pair of states $i, j$ an $n>0$ with $P_{i j}^{(n)}>0$. The entries $Q_{i k}$ of (2) of course represent the mean number of visits to $k$ of a path which starts at $i$.

We introduce some notation which will simplify the proof in $\$ 4$.

Let $\Omega$ be the set of nonnegative integer-valued sequences with coordinate functions $x(n, \omega)$. Assume $x(0, \omega) \equiv 0$. $B$ will be the smallest Borel field which contains the cylinder sets

$$
A\left(n_{1}, \cdots, n_{k} ; r_{1}, \cdots, r_{k}\right)=\left\{\omega \mid x\left(n_{j}, \omega\right)=r_{j} ; j=1, \cdots, k\right\} .
$$

The measure $P$ will be the extension of the measure $\mathcal{P}^{\prime}$ to $B$, where $\rho^{\prime}$ is defined on cylinder sets by

$$
\rho^{\prime}\left(A\left(n_{1}, \cdots, n_{k} ; r_{1}, \cdots, r_{k}\right)\right)=P_{0 r_{1}}^{\left(n_{1}\right)} P_{r_{1} r_{2}}^{\left(n_{2}-n_{1}\right)} \cdots P_{r_{k-1} r_{k}}^{\left(n_{k}-n_{k-1}\right)} .
$$

We recall for the reader's convenience a portion of the statement of the fundamental convergence theorem of Doob. (Cf. [2] for a complete exposition.)

Received by the editors September 11, 1962.

1 This research is sponsored by the United States Air Force under project RANDContract No. AF 49(638)-700-monitored by the Directorate of Development Planning, Deputy Chief of Staff, Research and Technology. Hq USAF.

2 The author wishes to thank T. E. Harris for posing this problem. 
Theorem. Let $P, Q$ be as in $\S 1$. For almost every $\omega \in \Omega$,

$$
\lim _{n \rightarrow \infty} \frac{Q_{i, x(n, \omega)}}{Q_{0, x(n, \omega)}}=q_{i}
$$

exists and satisfies

$$
\sum P_{i j} q_{j}=q_{i}
$$

This theorem will be necessary for the proof of Theorem 1 .

3. The necessary condition. Let $L_{k i}(j)$ denote the probability that a path starting at $k$, reaches $i$, with the first visit preceded by a visit to a state with index $\geqq j . L_{k i}(0)=L_{k i}$ is the probability of ever reaching $i$ from $k$. These quantities were introduced in [1] for $k \geqq j>i$.

THEOREM 1. Suppose there exists a non-negative solution to (1) which is not identically 0 . Then there is an infinite set $K$ of states such that

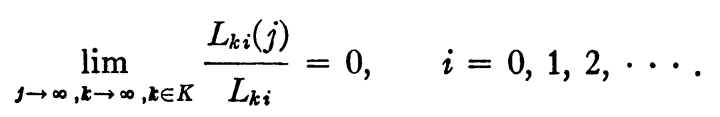

Proof. Fixing $i$, we will have for some $n>0, P_{0 i}^{(n)}>0$. Since $L_{k i} \geqq L_{k 0} P_{0 i}^{(n)}$ and $Q_{k 0}=L_{k 0}\left(1+Q_{00}\right)$, the inequality

$$
\frac{L_{k i}(j)}{L_{k i}} \leqq \frac{L_{k i}(j)}{Q_{k 0}} \frac{1+Q_{00}}{P_{0 i}^{(n)}}
$$

holds. It will therefore suffice to prove (5) for the ratios $L_{k i}(j) / Q_{k 0}$.

Secondly, let

$$
{ }_{i} P_{k j}^{(n)}=P_{r}\{x(n)=j ; x(m) \neq i, 0 \leqq m<n \mid x(0)=k\} .
$$

We could of course translate ${ }_{i} P_{k j}^{(n)}$ into the notation introduced in $\$ 2$, but this will not be necessary. What is essentially formula (4.2) in $[1]$ is the easily checked

$$
L_{k i}(j)=\sum_{r=j}^{\infty} \sum_{n=0}^{\infty}{ }_{i} P_{k r}^{(n)} P_{r i}
$$

Obviously, ${ }_{i} P_{k r}^{(n)} \leqq P_{k r}^{(n)}$, so

$$
L_{k i}(j) \leqq \sum_{r=j}^{\infty} \sum_{n=0}^{\infty} P_{k r}^{(n)} P_{r i}=\sum_{r=j}^{\infty} Q_{k r} P_{r i}
$$

For $k \neq i$,

$$
\sum_{r=0}^{\infty} Q_{k r} P_{r i}=Q_{k i}
$$


This well-known fact follows from the monotone convergence theorem applied to the partial sums for $Q$. From (6) and (7) we obtain for $k \neq i$,

$$
L_{k i}(j) \leqq Q_{k i}-\sum_{r=0}^{j-1} Q_{k r} P_{r i}
$$

Equation (4.10) in [1] evaluates the deficiency in (8) which will be unnecessary for our purposes.

Thirdly, let $a=\left\{a_{k}\right\}$ be the solution to (1) in the statement of our theorem. Since $a$ is not identically 0 , and for all $n, a P^{n}=a$, irreducibility implies $a_{k}>0$ for all $k$. We form a reverse chain, also transient, governed by the matrix $P^{*}$ below.

$$
P_{i j}^{*}=\frac{a_{j}}{a_{i}} P_{j i}, \quad Q_{i j}^{*}=\frac{a_{j}}{a_{i}} Q_{j i} .
$$

Substituting equations (9) into equations (3) and (4), Doob's theorem guarantees that for almost every $\omega \in \Omega^{*}$

$$
\liminf _{n \rightarrow \infty} \frac{Q_{x(n, \omega), i}}{Q_{x(n, \omega), 0}}=s_{i}, \quad i=0,1,2, \cdots
$$

exists and satisfies (1).

Fourthly, let $K$ be the set consisting of the states visited by any sample path for which (10) is satisfied. Arranging $K$ in increasing order will not affect the limit (10), due to the transience of the reverse chain.

Lastly, divide equation (8) by $Q_{k 0}$. We will show

$$
\lim _{g \rightarrow \infty, k \rightarrow \infty, k \in K}\left[\frac{Q_{k i}}{Q_{k 0}}-\sum_{r=0}^{j-1} \frac{Q_{k r}}{Q_{k 0}} P_{r i}\right]=0 .
$$

By (7) the difference in (11) is always nonnegative. Thus if (11) is false, there will be an $\epsilon>0$ and a sequence $\left(k_{n}, j_{n}\right), k_{n} \in K, k_{n}, j_{n} \rightarrow \infty$ such that

$$
\frac{Q_{k_{n}, i}}{Q_{k_{n}, 0}}-\sum_{r=0}^{j_{n}-1} \frac{Q_{k_{n}, r}}{Q_{k_{n}, 0}} P_{r i} \geqq \epsilon
$$

Let

$$
f_{n}(r)=\left\{\begin{array}{cc}
\frac{Q_{k_{n}, r}}{Q_{k_{n}, 0}}, & r<j_{n}, \\
0, & r \geqq j_{n},
\end{array}\right.
$$


be defined on the nonnegative integers $Z^{+}$. For $E \subset Z^{+}$define the measure $\mu_{i}(\cdot)$ by

By assumption,

$$
\mu_{i}(E)=\sum_{r \in E} P_{r i}
$$

$$
\begin{aligned}
\epsilon & \leqq \limsup _{n \rightarrow \infty}\left[f_{n}(i)-\int_{z^{+}} f_{n}(r) d \mu_{i}(r)\right] \\
& \leqq \lim _{n \rightarrow \infty} f_{n}(i)-\liminf _{n \rightarrow \infty} \int_{z^{+}} f_{n}(r) d \mu_{i}(r) \\
& \leqq \lim _{n \rightarrow \infty} f_{n}(i)-\int_{z^{+}} \lim _{n \rightarrow \infty} f_{n}(r) d \mu_{i}(r) \\
& =0 .
\end{aligned}
$$

The last inequality follows from Fatou's lemma. The contradiction thus obtained and inequality (8) give us the desired result.

\section{An unrelated result.}

Theorem 2. Let $P, Q$ be as in $\$ 1$ and $(\Omega, Q, P)$ as in $\$ 2$. For almost all $\omega \in \Omega$,

$$
\lim _{n \rightarrow \infty} \frac{P_{i, x(n, \omega)}}{Q_{i, x(n, \omega)}}=0, \quad i=0,1,2, \cdots .
$$

Proof. The functions

$$
g_{i, n}(\omega)=\frac{P_{i, x(n, \omega)}}{Q_{i, x(n, \omega)}}
$$

are measurable, as are the functions

$$
h_{i}(\omega)=\limsup _{n \rightarrow \infty} g_{i, n}(\omega)
$$

Let $A_{i}$ be the set of $\omega$ for which $h_{i}(\omega)>0$. If Theorem 2 were false, we would have for some $i, P\left(A_{i}\right)>0$. Since $P$ is countably additive, for $N$ sufficiently large $\odot\left(A_{i, N}\right)>0$ where $A_{i, N}$ is the set where $h_{i}(\omega)>1 / N$. To each $\omega \in A_{i, N}$ associate the set

$$
S(\omega)=\left\{x(n \omega) \mid \frac{P_{i, x(n, \omega)}}{Q_{i, x(n, \omega)}}>\frac{1}{N}\right\} .
$$

Note that each path $\omega$ visits $S(\omega)$ infinitely often. Let

$$
K=\bigcup_{\omega \in A_{i_{0} N}} S(\omega)
$$


For each $k \in K$,

$$
\frac{P_{i k}}{Q_{i k}}>\frac{1}{N} .
$$

This implies

$$
\sum_{k \in K} Q_{i k}<N \sum_{k \in K} P_{i k} \leqq N
$$

The sum on the left represents the mean number of times a path from $i$ visits the set $K$. There is a positive probability of a path from $i$ visiting 0 so the sum (14) for $i=0$ must also be finite. But each $\omega \in A_{i, N}$ visits $K$ infinitely often, and the assumption that $A_{i, N}$ has positive measure gives us a contradiction, proving the theorem.

From the proof follows the

Corollary. For almost all $\omega \in \Omega$, and all $i, j, k$

$$
\lim _{n \rightarrow \infty} \frac{P_{i, x(n, \omega)}^{(k)}}{Q_{j, x(n, \omega)}}=0 .
$$

\section{REFERENCES}

1. T. E. Harris, Transient Markov chains with stationary measures, Proc. Amer. Math. Soc. 8 (1957), 937-942.

2. G. A. Hunt, Markoff chains and Martin boundaries, Illinois J. Math. 4 (1960), 313-340.

The RAND CoRporation 